\title{
Estudio de la migración de aluminio desde las ollas a la colada de avena
}

\section{(Survey of migration of aluminium from pots to oat beverage)}

\author{
Tamara Fukalova Fukalova', Iván Tapia Calvopiña' ${ }^{1}$ Eva Quishpe ${ }^{1}$, Lorena Goetschel G. ${ }^{2}$, Wilson Parra ${ }^{1}$, \\ Vanessa Mena ${ }^{1}$
}

\begin{abstract}
Resumen
La migración de metales hacia los alimentos es un tema concerniente a la inocuidad alimentaria y la ingesta dietética es una fuente importante de exposición no ocupacional al aluminio. En esta investigación se determinó la cantidad de aluminio que se transfiere desde las ollas al preparar colada de avena con naranjilla (Solanum quitoense), bebida con pH ácido consumida ampliamente en Ecuador. Su preparación se realizó en ollas de aluminio de cinco marcas comercializadas en Quito y durante siete ciclos de cocción. La cuantificación de la concentración de aluminio transferido a las ciento cuarenta muestras de colada se realizó por espectroscopia de absorción atómica con llama de óxido nitroso-acetileno. Las concentraciones de aluminio transferido al alimento durante los siete tratamientos sobrepasan el límite permisible de $1 \mathrm{mg} \mathrm{Al} / \mathrm{kg}$ establecido por la Unión Europea (Reglamento Europeo UE 1416:2016), excepto para la marca de olla IV, que en el séptimo tratamiento presenta 0,76 $\pm 0,04 \mathrm{mg} \mathrm{Al} / \mathrm{kg}$. El análisis de varianza Anova de dos factores indica que las marcas de las ollas y el número de tratamientos aplicados sí afecta estadísticamente a la migración de aluminio.
\end{abstract}

\section{Palabras clave}

Migración, ollas de aluminio, absorción atómica, alimentos ácidos.

\begin{abstract}
The migration of metals from utensils to food during cooking is an issue of food safety. Dietary intake is the main source of non-occupational exposure to aluminum. This research allowed to know the amount of aluminum that is transferred when is preparing an oatmeal beverage with naranjilla (Solanum quitoense), an acidic pH drink, widely consumed in Ecuador. The beverage was prepared in aluminum pots of the five most commercialized brands in Quito and it was done in seven cooking cycles. The quantification of the concentration of transferred aluminum in the one hundred and forty oatmeal beverage was performed by atomic absorption Spectroscopy with nitrous oxideacetylene flame. The concentrations of aluminum transferred to the food during the seven treatments exceed the allowable limit of $1 \mathrm{mg} \mathrm{Al} / \mathrm{kg}$ established by the European Union (European Regulation EU 1416: 2016), except for the IV brand pot, which presents 0,76 $\pm 0,04 \mathrm{Al} / \mathrm{kg}$ in the seventh treatment. The two-factor Anova analysis of variance indicates that the brand of the pots and the number of treatments applied does affect aluminum migration.
\end{abstract}

\section{Keywords}

Migration, aluminium pots, atomic absorption, acid food

\section{Introducción}

El aluminio es un metal ampliamente distribuido en la naturaleza y utilizado para la elaboración de utensilios de cocina, como ollas, sartenes, recipientes o papel aluminio, empleados para preparar y conservar alimentos. Sin embargo, la preparación y almacenamiento de alimentos en recipientes de aluminio produce el aumento de contenido residual en los mismos (ELIKA, 2014). En los hogares ecuatorianos el uso de las ollas de aluminio es muy frecuente, al igual que en otros países, debido a su bajo costo y a su extensa distribución en el mercado. Los residuos de este metal al en-

1 Universidad Central del Ecuador, Quito - Ecuador ([tfukalova, iltapia, wparra, evaquishpe, vmmena]@uce.edu.ec).

2 Consultora independiente (loregoeagmail.com) 
contrarse en los alimentos pueden tener efectos tóxicos sobre la salud, ya que se ha sugerido que la exposición a este elemento se relaciona con enfermedades neurodegenerativas como el Alzheimer, la encefalopatía por diálisis y otras (Kawahara, Konoha, Nagata, \& Sadakane, 2007) (Suay \& Ballester, 2002). El resumen de salud pública del aluminio (Agencia para Sustancias Tóxicas y el Registro de Enfermedades de Estados Unidos, 2020), indica que las personas con enfermedades renales acumulan mayor cantidad de aluminio en sus cuerpos y son más susceptibles a desarrollar enfermedades óseas y cerebrales; aunque esta ficha toxicológica menciona que la asociación del aluminio con la enfermedad de Alzheimer (EA) aún no es certera. Otros autores mencionan que esta enfermedad neurodegenerativa se presenta como resultado de un deterioro multifactorial de origen genético y ambiental, y que la exposición al aluminio se encuentra dentro de los factores ambientales que podrían estar implicados en su desarrollo, de acuerdo a los resultados obtenidos en algunos estudios (Ferreira, Piai, Takayanagi, \& Segura-Muñoz, 2008), por lo que, se recomienda que se evite la exposición innecesaria a este metal, debido a su larga permanencia en el ser humano y a sus efectos en la salud (Kawahara, Konoha, Nagata, \& Sadakane, 2007).

Considerando que una de las vías de exposición al aluminio, son los alimentos preparados o almacenados en recipientes de aluminio, factores tales como pH del alimento, tiempo en el que permanece en contacto con el recipiente, consistencia de preparación y la mala práctica culinaria de guardar las comidas preparadas en las mismas ollas, aumentan la probabilidad de toxicidad por aluminio (Word Health Organization, 1997). La disolución de este metal, al igual que otros metales es altamente dependiente del $\mathrm{pH}$, temperatura y presencia de agentes complejantes. Al ser de oxidación fácil, la superficie de aluminio está cubierta de una película protectora compacta de óxido de aluminio. No obstante, la disolución de esta película protectora aumenta en medios ácidos y alcalinos, proceso que ocurre en la superficie de los utensilios de cocina de aluminio (Layla \& Juhaiman, 2010).

La normativa de la Unión Europea, en su Reglamento (UE) 2016/1416, del 24 de agosto de 2016, sobre materiales y objetos plásticos destinados a entrar en contacto con alimentos (Diario Oficial de la Unión Europea, 2016), fija como permitido un límite máximo de migración del aluminio de $1 \mathrm{mg} / \mathrm{kg}$ de alimento, mientras que el Comité del Codex Alimentario sobre Contaminantes de los Alimentos (JECFA) estableció una ingesta semanal tolerable provisional (ISTP) para el aluminio de $1 \mathrm{mg} / \mathrm{kg}$ peso (Codex Alimentarius, 2007).

A nivel mundial se han realizado varios estudios para conocer la cantidad de metal migrado a los alimentos desde los utensilios o recipientes de cocina. Así, Luján (2010) en Argentina, realizó la cocción de algunos alimentos, preparó infusiones e hirvió agua en recipientes de aluminio y otros materiales, y demostró que la transferencia del metal es más alta cuando el agua tiene pH ácido o neutro que cuando es alcalino. Concluye que: "todas las personas que cocinan el agua y los alimentos en recipientes de aluminio se exponen a dosis hasta ocho veces superiores al nivel máximo de aluminio que aporta el agua potable". Otro estudio realizado en España sobre los niveles de aluminio en varios alimentos consumidos en ese país, muestra concentraciones de aluminio entre 0.171 a 70.100 ug/g de alimento (López, Cabrera, Lorenzo, \& López, 2000). En una investigación realizada por Gramicconi, Ingrao, Milana et al. (1996), en Italia, los resultados muestran que la migración desde varios tipos de recipientes y ollas de aluminio hacia diferentes alimentos es variable, lo que puede deberse a parámetros como el pH, ya que alimentos como picles o salsa de tomate, que tienen $\mathrm{pH}$ ácido, presentan mayor migración.

Un estudio reciente llevado a cabo en Alemania por Stahl y colaboradores (2017), examinó la migración del aluminio desde recipientes de cocina, y desde los materiales de contacto hacia 
los alimentos y encontró que el límite máximo de transferencia de aluminio fijado en 5.00 mg/l por el Consejo Europeo en el 2013 (European Directorate for the Quality of Medicines \& HealthCare, 2013), no se excede en aceite o agua potable; sin embargo, el límite es claramente excedido en una solución al 0.5\% de ácido cítrico que llega a 638 mg/l. Esto significa que en la Ingesta Semanal Tolerable (TWl: $1 \mathrm{mg}$ Al/kg/semana), se excede en $298 \%$ para un niño de $15 \mathrm{~kg}$ y $63.8 \%$ para un adulto de $60 \mathrm{~kg}$ de peso. En India se investigó la migración de aluminio utilizando ollas nuevas de este metal y se observó que a medida que el pH baja y que se incrementa el uso de las ollas, disminuye la concentración de aluminio y que la migración es muy baja cuando se utiliza utensilios de aluminio anodizado (Semwal, Padmashree, Khan, Sharma, \& Bawa, 2006).

En Ecuador se realizó un estudio donde se utilizaron ollas nuevas de las mismas marcas que en el presente estudio. Se cocinó arroz, alimento de pH neutro, y se obtuvieron resultados dentro de la normativa europea a partir del tercer ciclo de cocción (Cisneros, Tapia, Goetschel, \& Fukalova, 2019). Además, se cuenta con resultados de una investigación en la que se analizó el contenido de aluminio en muestras de colada morada, bebida de pH ácido, comercializada en mercados, y se observó que la mayoría de las muestras presenta niveles de aluminio superiores al fijado en la normativa (Salazar \& Goetschel, 2018).

El objetivo del presente estudio fue determinar la concentración del residuo de aluminio migrado desde las ollas de este metal, en la preparación de colada de avena con naranjilla, por espectroscopia de absorción atómica, a fin de obtener datos de línea base y contrastarlos con el límite de migración permitido para este metal a los alimentos, establecido por la Unión Europea (UE). Este alimento fue escogido por ser una bebida tradicional consumida en Ecuador, que generalmente se prepara con frutas, por lo que presenta un pH ácido. En el año 2018, el Ecuador fue el mayor consumidor de avena para alimentación humana en América del Sur (Ministerio de Agricultura de Chile Oficina de Estudios y Políticas Agrarias, 2018), y gran parte de este alimento se utiliza para elaborar colada, lo que justifica la importancia de realizar este estudio.

\section{Metodología}

\subsection{Selección de los recipientes de aluminio}

Se seleccionaron cinco marcas de ollas de aluminio para preparación de la colada de avena, a un rango de pH determinado y la misma cantidad de ingredientes durante siete ciclos de cocción.

Las marcas adquiridas se delimitaron por procesamiento de la información recolectada a través de una encuesta realizada a 100 usuarios in situ (un muestreo aleatorio simple sin reposición), en tres sectores muy concurridos de la ciudad de Quito. Se adquirieron las ollas de las marcas de uso más frecuente según las encuestas y de características similares en lo que al volumen se refiere. Se las codificó como I, II, III, IV, V y VI; se midió el espesor de cada olla con un micrómetro análogo marca Mitutoyo (precisión $\pm 2 \mu \mathrm{m}$ ) y se realizó el trabajo experimental.

\subsection{Preparación de la unidad experimental}

Se estableció una formulación de colada de avena con naranjilla, precisando las cantidades de ingredientes (avena, azúcar, pulpa de naranjilla y agua potable), para mantener el pH en el rango de 4.0 - 4.5. Para la preparación de la colada, se colocó en cada olla $600 \mathrm{ml}$ de agua de calidad para consumo humano y uso doméstico con las siguientes características: pH entre 6.5 y 8.5; dureza 300 mg/l y contenido máximo de aluminio 0.2 mg/l. Se llevó a ebullición, luego se colo- 
caron $25 \mathrm{~g}$ de avena, $25 \mathrm{ml}$ de pulpa de naranjilla y $25 \mathrm{~g}$ de azúcar (de una sola marca comercial conocida). Se dejó hervir por 15 minutos, revolviendo cada 5 minutos por 30 segundos, hasta obtener una consistencia espesa. Se retiró del fuego y se dejó enfriar.

\subsection{Concentración de aluminio migrado}

Para descartar el aporte de aluminio como fuente indirecta, los ingredientes de la colada y el agua utilizados en la cocción fueron analizados previamente por espectroscopia de absorción atómica, obteniendo resultados por debajo del límite de detección $<0.12 \mathrm{mg} / \mathrm{l}$ de aluminio en todos los análisis efectuados.

En total 140 muestras fueron analizadas. De cada unidad experimental se tomaron 50.0 gramos de colada de avena por cada ciclo de cocción. Esta muestra se calcinó en una mufla Carbolite, modelo RHF 1400 para obtener cenizas totales (método oficial AOAC 923.03). A continuación, éstas se sometieron a un proceso de digestión ácida, añadiendo $2 \mathrm{ml}$ de ácido nítrico (65\% Emsurer) y 2 $\mathrm{ml}$ de peróxido de hidrógeno (30 \% Emsurer) y un proceso de calentamiento hasta que quede un residuo cercano a $3 \mathrm{ml}$. Las muestras digeridas se filtraron y se diluyeron a $10 \mathrm{ml}$ con agua destilada.

La concentración de aluminio se determinó mediante la técnica de espectroscopia de absorción atómica (AAS), en un equipo Perkin Elmer, modelo AA 240FS con comburente óxido nitroso-acetileno y longitud de onda $309.30 \mathrm{~nm}$.

La cuantificación se realizó mediante el uso de una curva de calibración en el rango de 2 a 10 mg/l, a partir de un estándar de aluminio de 1000 mg/l (AcuStandar, lote 216115074). Para aseguramiento de la calidad se verificó la linealidad, exactitud y precisión del método, comprobando los límites de detección (LD), de cuantificación (LC), porcentaje de recuperación y la repetibilidad.

\subsection{Análisis estadístico}

Para determinar la migración de aluminio, se trabajó con cinco marcas de ollas en siete ciclos de cocción. Se realizó un análisis de varianza de dos factores con una sola réplica por muestra para un total de 140 unidades experimentales. Los resultados se analizaron con el programa IBM SPSS Statistic versión 20.

\subsection{Estudio de microsuperficie de ollas}

Se utilizó un microscopio de fuerza atómica (AFM) marca Park System modelo NX10, Cantilibre NCHR 10 M, con punto fijo 9E3 (nm), amplitud 20.185E3 (nm), frecuencia 308.08E3 (Hz) y conducción $0.64 \%$, para determinar la rugosidad en un área de $10 \mu \mathrm{m}$ de la olla de la marca I, por ser la de mayor preferencia en el mercado, luego del primer uso y de siete usos, lo que permitió evaluar la diferencia de rugosidad encontrada.

\section{Resultados y discusión}

\subsection{Identificación de las marcas de ollas para la investigación}

Según resultados procesados de la encuesta realizada (análisis estadístico MODA), en la figura 1 se muestra la preferencia de las marcas de ollas en la ciudad de Quito. La investigación se realizó con las cinco marcas de mayor aceptación en el mercado. 
Figura 1. Porcentaje de las ollas más adquiridas en una encuesta realizada en la ciudad de Quito

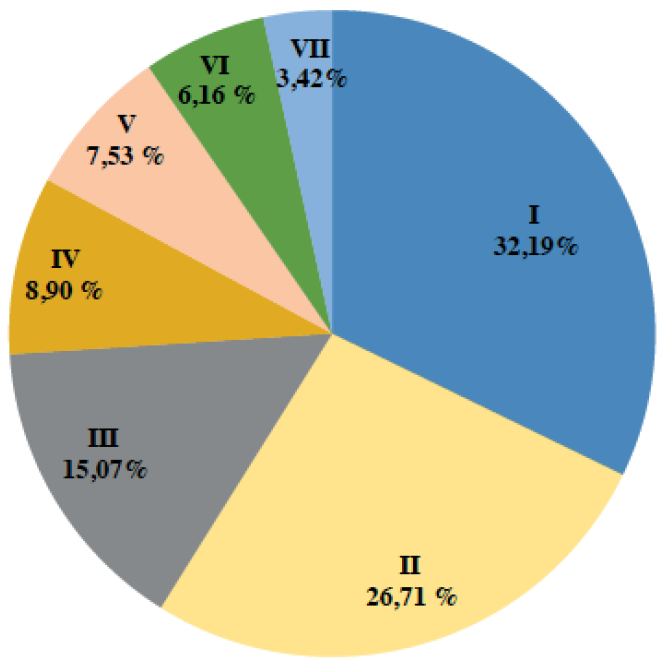

La preferencia de las marcas de ollas se debe básicamente al precio y a la calidad (durabilidad), tal como se refleja en la figura 2. Además, para los encuestados hay predilección por el espesor de las paredes de las ollas a utilizar para la elaboración de los preparados líquidos, como es la colada de avena, lo que se muestra en la figura 3.

Figura 2. Criterios de preferencia de las ollas

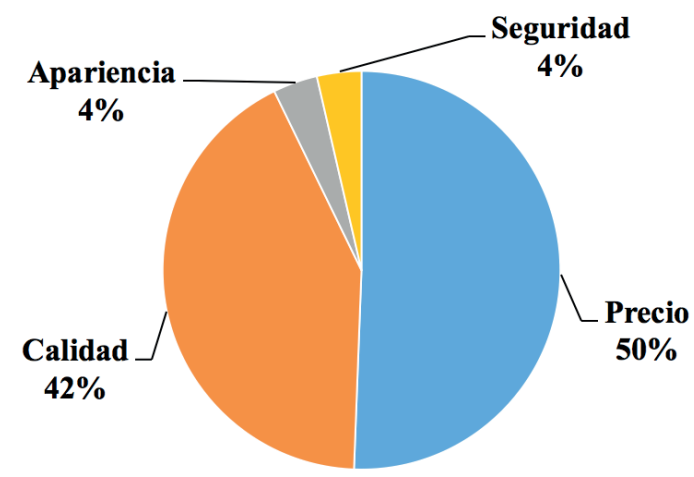

Figura 3. Predilección por el espesor de las ollas

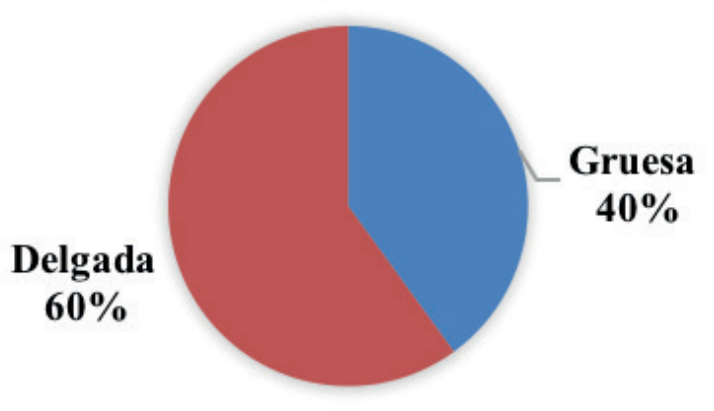

\subsection{Determinación del espesor de las paredes de las ollas}

Como se muestra en la tabla N1, el espesor de las cinco marcas de ollas utilizadas en la investigación presentó valores entre $0.51 \pm 0.03$ y $1.22 \pm 0.04 \mathrm{~mm}$, aceptables según la normativa ecuatoriana INEN 2361:2005 (Instituto Ecuatoriano de Normalización, 2012), para ollas de diámetro nominal de $16 \mathrm{~cm}$, la que indica que el espesor mínimo de la pared es $0.5 \mathrm{~mm}$. 
Tabla 1. Resultados de espesor de las ollas de aluminio

\begin{tabular}{|c|c|c|c|c|c|}
\hline \multicolumn{7}{|c|}{ Marcas de ollas } \\
\hline Repeticiones & I & II & III & IV & V \\
\hline \multicolumn{7}{|c|}{ Medida del espesor (mm) } \\
\hline 1 & 1.10 & 1.19 & 0.51 & 0.84 & 1.22 \\
\hline 2 & 1.06 & 1.21 & 0.52 & 0.82 & 1.23 \\
\hline 3 & 1.09 & 1.20 & 0.50 & 0.84 & 1,20 \\
\hline Promedio & 1.08 & 1.20 & 0.51 & 0.83 & 1,22 \\
\hline Desviación & 0.021 & 0.010 & 0.010 & 0.012 & 0.015 \\
\hline LC al 95\% & $1.08 \pm 0.05$ & $1.20 \pm 0.03$ & $0.51 \pm 0.03$ & $0.83 \pm 0.03$ & $1.22 \pm 0.04$ \\
\hline
\end{tabular}

\subsection{Estandarización del método de análisis por absorción atómica}

Se cumplieron los parámetros de verificación del método directo de llama de óxido nitroso-acetileno 3.20 APHA 3111 D, para una muestra de alimento de características ácidas (colada de avena con naranjilla), como linealidad, exactitud, precisión del método. La curva de calibración presentó un alto coeficiente de correlación lineal $(R>0.9993)$ y se estableció que el límite de detección (LD) es de 0.12 mg/l, y el límite de cuantificación (LC) es de 0.4 mg/l. La exactitud del método evidenció $97.95 \%$ de recuperación, que se encuentra dentro del rango validado para el equipo que es 80-120 \%. El ensayo de precisión permitió asegurar la repetibilidad del método, con coeficiente de variación con un promedio de $0.44 \%$.

\section{Concentraciones de aluminio de las muestras analizadas}

En la tabla N2 se encuentran los promedios de la concentración de aluminio migrado hacia las muestras de colada, alimento ácido, cuyo pH varió de 4.30 a 4.40 y sus grados Brix de 6.5 a 7.2. Los valores máximos de $2.40 \pm 0.03 \mathrm{mg} / \mathrm{kg}$ corresponden al primer tratamiento en la marca $\vee$ y valores mínimos de $0.76 \pm 0.04 \mathrm{mg} / \mathrm{kg}$ al séptimo tratamiento en la marca IV. Se observó que las ollas, durante los siete ciclos de cocción aplicados para la colada, presentaron una migración de aluminio por encima del límite permitido por la Unión Europea (UE), excepto en el último tratamiento para la marca IV (0.76 mg Al/ $\mathrm{kg})$.

Tabla 2. Resultados de análisis de aluminio migrado para cinco muestras de olla y siete ciclos de cocción

\begin{tabular}{|c|c|c|c|c|c|}
\hline \multirow{2}{*}{ Ciclos de cocción } & \multicolumn{5}{|c|}{ Marcas de ollas utilizada } \\
\cline { 2 - 6 } & I & II & III & IV & V \\
\cline { 2 - 6 } & \multicolumn{5}{|c|}{ Concentración del Al (mg/kg) } \\
\hline $\mathbf{1}^{\circ}$ & $2.13 \pm 0.07$ & $1.45 \pm 0.04$ & $1.84 \pm 0.04$ & $2.03 \pm 0.02$ & $2.40 \pm 0.03$ \\
\hline $2^{\circ}$ & $1.53 \pm 0.02$ & $1.38 \pm 0.01$ & $1.75 \pm 0.02$ & $1.66 \pm 0.02$ & $2.25 \pm 0.01$ \\
\hline $3^{\circ}$ & $1.54 \pm 0.05$ & $1.34 \pm 0.02$ & $1.46 \pm 0.04$ & $1.43 \pm 0.03$ & $1.69 \pm 0.06$ \\
\hline $4^{\circ}$ & $1.26 \pm 0.07$ & $1.21 \pm 0.03$ & $1.40 \pm 0.01$ & $1.22 \pm 0.02$ & $1.47 \pm 0.02$ \\
\hline $5^{\circ}$ & $1.33 \pm 0.01$ & $1.07 \pm 0.03$ & $1.28 \pm 0.01$ & $1.17 \pm 0.02$ & $1.40 \pm 0.02$ \\
\hline $6^{\circ}$ & $1.22 \pm 0.04$ & $1.09 \pm 0.01$ & $1.04 \pm 0.01$ & $1.13 \pm 0.03$ & $1.39 \pm 0.01$ \\
\hline $7^{\circ}$ & $1.18 \pm 0.02$ & $1.02 \pm 0.03$ & $1.05 \pm 0.04$ & $0.76 \pm 0.04$ & $1.23 \pm 0.02$ \\
\hline
\end{tabular}


En el análisis de varianza de dos factores se observa que los ciclos de cocción, al igual que las marcas de ollas, tienen un efecto significativo, al $95 \%$ de confianza, en la cantidad de aluminio migrado durante la cocción de la colada de avena. Adicionalmente, al aplicar una prueba múltiple (DMS), se concluye que el comportamiento de la migración de aluminio hacia las muestras, en las cinco marcas de ollas durante los siete ciclos de cocción, disminuye conforme aumenta el número de ciclos de cocción.

En la investigación realizada por (Salazar \& Goetschel, 2018), en muestras preparadas de colada morada, cuyo pH osciló entre 3.72 y 4.58, adquiridas en los mercados de Quito, se determinó que la migración de aluminio, durante el almacenamiento del alimento en ollas del mismo metal, sobrepasa el límite permisible establecido por la UE. Los parámetros de preparación y cocción de estas muestras fueron desconocidos, mientras que este estudio se realizó bajo condiciones controladas y el pH fluctúa entre 4.3 y 4.4. En los dos estudios, la concentración de aluminio sobrepasa los límites establecidos por la UE.

En el trabajo realizado por (Cisneros, Tapia, Goetschel, \& Fukalova, 2019), se analizó la migración de aluminio en las mismas marcas de ollas en seis ciclos de cocción, pero con un alimento de pH neutro que es el arroz. La migración de aluminio fue detectable hasta el tercer ciclo y en los últimos ciclos fue menor al límite máximo establecido por la UE que es $1 \mathrm{mg} / \mathrm{l}$. Estos resultados contrastan con los obtenidos en este estudio y permiten determinar la influencia del pH en la migración del metal.

Al comparar los resultados de este estudio con los resultados obtenidos en otros países (Gramiccioni, Ingrao, Milana, Santaroni, \& Tomassi, 1996). (Luján, 2010), se observa semejanza en cuanto a que la migración a pH ácido es mayor que a pH neutro o básico, lo que concuerda también con el estudio en el cual la migración es mayor en la solución de ácido cítrico al 0.5\%, cuyo pH es menor a 4.5 (Stahl, y otros, 2017). Mientras que los resultados obtenidos por López, Cabrera y Lorenzo (2000) en España, muestran los siguientes valores: 1.362 a $6.610 \mu \mathrm{g} / \mathrm{g}$ en alimentos del mar; 0.171 a $29.688 \mu \mathrm{g} / \mathrm{g}$ en vegetales; 19.560 a $70.100 \mu \mathrm{g} / \mathrm{g}$ en aceite de oliva; 0.424 a $6.430 \mu \mathrm{g} / \mathrm{g}$ en lácteos; y 25.600 a $58.057 \mu \mathrm{g} / \mathrm{g}$ en bebidas estimulantes e infusiones, mostrando una concentración variable que puede estar relacionada al pH del alimento.

Los resultados corroboran lo concluido por Semwal, Padmashree, Khan et al. (2006). ya que, al utilizar ollas nuevas, la concentración de aluminio migrado disminuye a medida que se incrementa el uso de las ollas. También concluyen que los alimentos ácidos aumentan la migración del metal. En ese estudio adicionalmente se realizaron pruebas con utensilios de aluminio anodizado, que mostró menor migración, por lo que sería conveniente estudiar los materiales de cada una de las marcas de ollas de aluminio comercializadas en Ecuador y relacionarlo con la migración, lo que podría explicar las variaciones en la cantidad de aluminio migrada; por ejemplo, en este caso, en la marca IV, que incluso llega a ser menor del límite establecido a partir del séptimo ciclo.

\section{Evaluación de la rugosidad de la olla tomada como referencia}

El análisis de rugosidad de la olla marca I, que es la de mayor comercialización en la ciudad de Quito según las encuestas, se realizó comparando los valores para el fragmento de la olla de un solo uso y luego de siete usos. Los resultados se muestran en la tabla 3, e indican que la rugosidad obtenida en un área de $10 \mathrm{um}^{2}$ en la olla de un solo uso, es mayor y disminuye cuando se aplican varios usos según se visualiza en las figuras 5 y 6. 
Tabla 3. Rugosidad obtenida en la olla I

\begin{tabular}{|l|l|l|l|l|}
\hline \multirow{2}{*}{ Muestra de olla I } & \multicolumn{3}{|c|}{ Ra } & \multicolumn{1}{c|}{ Rz } \\
\cline { 2 - 5 } & \multicolumn{1}{|c|}{$\mathrm{Nm}$} & $\mu \mathrm{m}$ & \multicolumn{1}{c|}{$\mathrm{Nm}$} \\
\hline Un solo uso & 55.909 & 0.056 & 530.769 & 0.531 \\
\hline Luego de siete usos & 34.928 & 0.035 & 400.410 & 0.400 \\
\hline $\begin{array}{l}\text { Rz: media de los valores absolutos (5 crestas perfil más alto y 5 valles del perfil más bajo). } \\
\text { Ra: media aritmética de los valores absolutos de las separaciones y del perfil rugoso. }\end{array}$ \\
\hline
\end{tabular}

Figura 5. Rugosidad de la olla I de un solo uso determinada mediante AFM

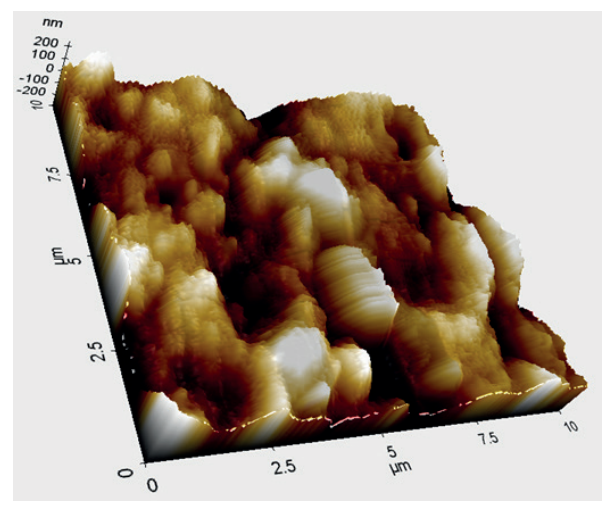

Figura 6. Rugosidad final de la olla luego de siete usos determinada mediante AFM

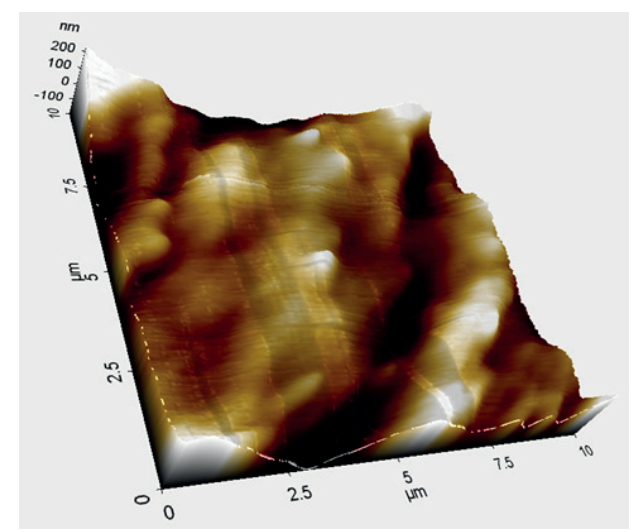

Las imágenes permiten observar el cambio de la rugosidad de la superficie de la olla I. En el séptimo uso se evidencia la disminución de las crestas y valles que constituyen la rugosidad, obteniendo un Ra experimental que corresponde a la superficie de calibre de un grado de rugosidad N2, según la norma DIN 4769 y de acuerdo al documento sobre Rugosidad Superficial (Grupo Tecnología Médica, 2017), en donde también se explica que el perfil de rugosidad o de textura primaria depende de los procesos de mecanizado durante la fabricación. La rugosidad superficial de la olla I disminuyó luego del uso, lo que puede relacionarse con la cantidad de aluminio desprendido desde la superficie. El descenso en la lixiviación de aluminio desde la olla IV hacia la colada de avena en el último ciclo de cocción, por debajo del límite permitido, podría 
relacionarse con una insignificante pérdida de su rugosidad en la micro superficie de la olla, análisis que no se realizó en este estudio.

La variabilidad en los resultados obtenidos demuestra la posible influencia de la calidad del material de las ollas, además del proceso de fabricación. Ya que según otros estudios solo el aluminio anodizado o recubierto aporta una capa protectora que evita que el metal se transfiera al alimento (Vergara, Nerey, \& Guédez, 2011) y puede resistir la corrosión ocasionada por el agua, la sal y otros agentes (Gupta, Meenu, \& Peshin, 2019).

\section{Conclusiones}

Los resultados obtenidos en el presente estudio permitieron conocer las concentraciones de aluminio migrado hacia la colada de avena, debido al uso de las ollas de aluminio con mayor incidencia de comercialización en Quito (Ecuador), y se evidenció que las concentraciones están por encima del nivel máximo permitido por la normativa de la UE para este elemento.

La cantidad de aluminio migrado hacia las muestras, indica que los alimentos ácidos preparados en recipientes de aluminio son contaminados con trazas de este metal, lo que puede representar riesgos para la salud de los consumidores. Los resultados indican que la concentración en promedio de aluminio encontrado durante siete ciclos de cocción para cuatro ollas, supera el límite máximo establecido por el Reglamento Europeo UE 1416:2016 (1 mg/kg de alimento).

El contenido de aluminio de los distintos alimentos depende no solamente de sus características intrínsecas sino también de la forma en la fueron preparados, por lo tanto, los hábitos alimentarios de una población condicionan fuertemente la ingesta diaria de aluminio. Es el caso de adicionarle una fruta ácida como la naranjilla a una bebida de avena.

El uso diario de utensilios y cacerolas de aluminio no es recomendado para la cocción de alimentos ácidos, ni para almacenar los alimentos en recipientes de aluminio, ya que el pH bajo (alta acidez) afecta la rugosidad de la micro superficie de las ollas, que trasladan trazas de este metal a los alimentos.

\section{Bibliografía}

Agencia para Sustancias Tóxicas y el Registro de Enfermedades de Estados Unidos. (2020). Atsdr. Recuperado de https://www.atsdr.cdc.gov/es/toxfaqs/es_tfacts22.html

Cisneros, K.; Tapia, I.; Goetschel, L. y Fukalova. T. (2019). Evaluación de migración de aluminio durante la cocción de arroz blanco en ollas de aluminio. Info Analítica Vol. 7 No. 2, 57-69.

Codex Alimentarius. (2007). Informe del comité del codex sobre contaminantes de los alimentos. Beijing: Codex.

Diario Oficial de la Unión Europea. (2016). Reglamento (UE) 2016/1416 de la Comisión de la Unión Europea sobre materiales y objetos plásticos destinados a entrar en contacto con alimento.

Elika. (2014). Aluminio. Fundación Vasca para la Seguridad Agroalimentaria. Recuperado de https://seguridadalimentaria.elika.eus/wp-content/uploads/2018/01/29.Aluminio.pdf

European Directorate for the Quality of Medicines \& HealthCare. (2013). Metals and Alloys Used in Food Contact Materials and Articles. Francia: Council of Europe.

Ferreira, P.; Piai. K.; Takayanagi, A. y Segura-Muñoz, S. (2008). Aluminio como factor de riesgo para la enfermedad de Alzheimer. Revista Latino Americana de Enfermagem. Vol.16(1), 151-157.

Gramiccioni, L.; Ingrao, G.; Milana, M. et al. (1996). Aluminium levels in Italian Diets and in Selected Foods from Aluminium Utensils. Food Additives \& Contaminants. 767-774. 
Grupo Tecnología Médica. (2017). Mitutoyo Rugosidad Superficial. Catálogo número E70 Recuperado el 18 de mayo de 2019 de http://www3.fi.mdp.edu.ar/tecnologia/archivos/TecFab/10.pdf

Gupta, Y.; Meenu, M. y Peshin, S. (2019). Aluminium Utensils: Is it a Concern? The National Medical Journal of India. 38-40.

Instituto Ecuatoriano de Normalización. (2012). NTE INEN 2361:2005 Norma Técnica Ecuatoriana: Artículos de uso doméstico. Ollas de aluminio. Quito- Ecuador: INEN.

Kawahara, M.; Konoha, K.; Nagata, T. y Sadakane, Y. (2007). Aluminum and Human Health: Its Intake. Bioavailability. Biomed Res Trace Elements. 211-220.

Layla, A. y Juhaiman, A. (2010). Estimating Aluminium Leaching from Aluminium Cook Wares in Different Meat Extract and Milk. Journal of Saudi Chemical Society. 131-137.

López, F.; Cabrera, C.; Lorenzo, M. y López, M. (2000). Aluminum Content in Foods and Beverages Consumed in the Spanish Diet. Journal of Food Science Vol. 2. 206-210.

Luján, J. (2010). Ingesta de aluminio al cocinar alimentos y hervir agua con utensilios domésticos. Tecnología y ciencia. Año 3- No. 6. 26-32.

Ministerio de Agricultura de Chile Oficina de Estudios y Políticas Agrarias. (diciembre de 2018). Prospectivas del mercado mundial de la avena para consumo humano. Recuperado de Odepa: https:// www.odepa.gob.cl/wp-content/uploads/2018/12/estudioAvena2018.pdf

Rugosidad, S. (2010). Recuperado de Grupo tecnología mecánica-procesos de fabricacion: www.academia.edu.ar; www.3.fi.mdp.edu.ar/tecnologia/archivos/TecFab/10.pdf

Salazar, N. y Goetschel, L. (2018). Determinación de aluminio en muestras de colada morada, mediante el método de absorción atómica-horno de grafito. Quito: Universidad Central del Ecuador.

Semwal, A.; Padmashree, A.; Khan, M. et al. (2006). Leaching of Aluminium from Utensils during Cooking of Food. Journal of the Science of Food and Agriculture 86(14). 2425 - 2430.

Stahl, T.; Falk, S.; Rohrbeck, A. et al. (2017). Migration of Aluminium from Food Contact Materials to Food-a Health Risk for Consumers? Environmental Sciences Europe. 29(19). 1-8.

Suay, L. y Ballester, F. (2002). Revisión de los estudios sobre exposición al aluminio y enfermedad de Alzheimer. Revista Española de Salud Pública. 6(76). 645-658.

Vergara, L.; Nerey, L. y Guédez, V. (2011). Modelo predictivo de la rugosidad y porosidad en aluminio Al3003B14 y Al6063-T6 anodizado usando análisis multi-factorial. Rev Ciencia e Ingenieria. 105-112.

Word Health Organization. (1997). International Proramme on Chemical Safety. Environmental Criteria 194: Aluminium. Geneve: United Nations Environment Programm. 